\title{
Orbital selective and tunable Kondo effect of magnetic adatoms on graphene: Correlated electronic structure calculations
}

\author{
D. Jacob \\ Max-Planck-Institut für Mikrostrukturphysik, Weinberg 2, 06120 Halle, Germany \\ G. Kotliar \\ Dept. of Physics 8 Astronomy, Rutgers University, \\ 136 Frelinghuysen Rd., Piscataway, NJ 08854, USA
}

(Dated: July 14, 2018)

\begin{abstract}
We have studied the effect of dynamical correlations on the electronic structure of single Co adatoms on graphene monolayers with a recently developed novel method for nanoscopic materials that combines density functional calculations with a fully dynamical treatment of the strongly interacting $3 d$-electrons. The coupling of the Co $3 d$-shell to the graphene substrate and hence the dynamic correlations are strongly dependent on the orbital symmetry and the system parameters (temperature, distance of the adatom from the graphene sheet, gate voltage). When the Kondo effect takes place, we find that the dynamical correlations give rise to strongly temperature-dependent peaks in the Co $3 d$-spectra near the Fermi level. Moreover, we find that the Kondo effect can be tuned by the application of a gate voltage. It turns out that the position of the Kondo peaks is pinned to the Dirac points of graphene rather than to the chemical potential.
\end{abstract}

PACS numbers:

\section{INTRODUCTION}

Since its recent discovery, Graphene - a monolayer of graphite - has become the subject of intense research due to its peculiar electronic properties $\underline{1-4}$. The unusual properties of graphene are largely a result of the linear dispersion of electron bands at low energies and its twodimensionality. This allows to describe the charge carriers in graphene as a two-dimensional gas of massless Dirac-fermions. The unusual electronic properties and the fact that its chemical potential can easily be tuned by a gate voltage make graphene an ideal experimental testing ground for exotic physics as well as a promising basis for novel nano-electronics devices. For recent reviews of this rapidly developing field and additional references, see e.g. Refs. 5 - 7 .

Graphene is also a good testing ground for exotic Kondo physics ${ }^{8}$ : First, the linearly vanishing DOS of graphene favours the formation of a local moment $\mathrm{g}^{\frac{9}{}}$ which is a necessary condition for Kondo physics. Also vacancies and edge defects in graphene have been predicted to give rise to magnetic moments 10,11 . Very recently, the Kondo effect has actually been observed at local defects in graphene providing evidence that defects in graphene are indeed magnetic $\frac{12}{2}$. On the other hand, as a result of the linearly vanishing DOS, a magnetic moment coupled to a graphene layer can only display a Kondo effect if the strength of the coupling is strong enough $13-15$. Furthermore because of the existence of two inequivalent Dirac cones, it has multiple channels which can in principle screen the impurity spin and could therefore lead to a multichannel Kondo effect and hence non-fermi liquid behaviour $\underline{16}-\underline{20}$. Kondo effect and the possibilty of multichannel Kondo effect in graphene have been discussed in a number of recent publications $21-26$.
Moreover, it is possible to probe the Kondo effect of magnetic adatoms on graphene by scanning tunneling microscopy (STM). Similar to STM spectroscopy of magnetic adatoms and molecules on metallic surfaces $27-30$, the Kondo effect could show up as Fano lineshapes in the conductance vs. bias voltage characteristics of the STM or as additional temperature dependent peaks in the STM spectra. But in comparison with normal metallic surfaces the Fano lineshapes for graphene are expected to be more asymmetric and strongly dependend on the symmetry of the $d$-orbitals of magnetic atom 25 .

The goal of this work is to perform a realistic study of the Kondo effect for a single Co adatom on an otherwise perfect graphene sheet by using a recently developed approach for nanoscopic systems that combines ab initio electronic structure calculations on the level of density functional theory (DFT) calculations with a full dynamical treatment of the strongly interacting $3 d$-electrons 31 . A realistic treatment allows us to investigate under which conditions the Kondo effect takes place in a given orbital and the detailed spectral functions that arise from the Kondo effect in a semi-metallic substrate.

This paper is organized as follows: In Sec. II we briefly describe our recently developed GGA+OCA method ${ }^{31}$ for the quasi $a b$ initio description of the electronic structure of nanoscopic systems fully taking into account dynamical correlations by combining DFT calculations with a dynamical treatment of the strongly interacting $3 d$-electrons in the so-called one-crossing approximation (OCA).

In Sec. [II we present DFT electronic structure calculations in the generalized gradient approximation (GGA) for determining the most favourable adsorption site for the Co atom on the graphene sheet. In agreement with previous results by other groups ${ }^{24,25,39}$ we find that the 
hollow site is the most favourable adsorption site. Using hybrid functional calculations which generally predict better geometries for molecules we have then optimized the adsorption height of the Co atom at the hollow site of the graphene. Using GGA we have also calculated the charge and spin of the Co atom in dependence of the distance to the graphene sheet for the three different adsorption sites. The plots indicate that depending on the distance the Co atom can be either in the local moment regime or in weak correlation regime. Since the potential energy curves are very shallow it seems feasible that the correlation regime be controlled via the distance of the Co atom to the graphene substrate in an actual experiment.

In Sec. IV we study the impact of dynamical correlations of the Co $3 d$-electrons on the elctronic structure of the Co atom and the graphene sheet using the GGA+OCA method for nanoscopic systems as described in Sec. II. We find that the dynamical correlations of the Co $3 d$-electrons give rise to strongly temperaturedependent peaks near the Fermi level in the spectral density of the Co $3 d$-shell but also in the spectral density of the $p_{z}$-orbitals of nearby carbon atoms. Furthermore, we find that the Co $3 d$-spectra depend quite strongly on the exact energies of the Co $3 d$-levels. Accordingly, the application of a gate voltage which changes the chemical potential of the graphene substrate has a similar effect on the spectra. Hence the dynamic correlations and the Kondo effect can be controlled by the application of a gate voltage.

Finally, in Sec. $\nabla$ we conclude this work with a general discussion of the results in the light of Kondo physics.

\section{GGA+OCA METHOD}

We consider a single Co adatom adsorbed at the hollow site of an otherwise perfect graphene sheet as shown in Fig. 3. To proceed we divide the system into two parts. The central region $\mathrm{C}$ which contains the Co adatom and a number of carbon atoms is embeded into the host material $\mathrm{H}$ given by a perfect graphene sheet. The central region $\mathrm{C}$ has to be chosen big enough so that the electronic structure outside of the region can be assumed to be that of a perfect graphene sheet.

As a first step a mean-field description of the electronic structure of the system is obtained from DFT calculations on the level of GGA using the CRYSTAL06 ab initio electronic structure code $\mathrm{e}^{32}$ together with the 6-31G gaussian basis set: By setting up a 2D lattice of supercells of the $\mathrm{C}$ region we obtain an effective one-body Hamiltonian $\mathbf{H}_{\mathrm{C}}$ for the $\mathrm{C}$ region from the Kohn-Sham Hamiltonian of the converged GGA calculation. Analogously, we obtain an effective mean-field description of the host material from a GGA calculation for a supercell of clean graphene corresponding to the $\mathrm{C}$ region.

The Green's function of the central unit cell $\mathrm{C}$ is then given by:

$$
\mathbf{G}_{\mathrm{C}}(\omega)=\left(\omega+\mu-\mathbf{H}_{\mathrm{C}}+\mathbf{h}_{\mathrm{dcc}}-\Sigma_{3 d}(\omega)-\boldsymbol{\Sigma}_{\mathrm{H}}(\omega)\right)^{-1}
$$

$\boldsymbol{\Sigma}_{\mathrm{H}}(\omega)$ is the embedding self-energy which describes the dynamic hybridization between the central region $\mathrm{C}$ and the host material H. The embedding self-energy is obtained from the GGA calculation of the perfect graphene sheet as explained in App. A.

$\Sigma_{3 d}(\omega)$ is the electronic self-energy describing the dynamic correlations of the strongly interacting $3 d$ electrons. The strong interactions of the Co $3 d$-shell are captured by adding a Hubbard-like interaction term to the one-body Hamiltonian within the correlated $3 d-$ subspace:

$$
\hat{\mathcal{H}}_{U}=\frac{1}{2} \sum_{\substack{i j k l \\ \sigma_{1} \sigma_{2}}} U_{i j k l} \hat{d}_{i \sigma_{1}}^{\dagger} \hat{d}_{j \sigma_{2}}^{\dagger} \hat{d}_{l \sigma_{2}} \hat{d}_{k \sigma_{1}}
$$

$U_{i j k l}$ are the matrix elements of the effective Coulomb interaction of the $3 d$-electrons which is different from the bare Coulomb interaction due to the screening by the conduction electrons. Here we take a model interaction taking into account only the direct Coulomb repulsion $U$ between electrons and a Hund's rule coupling $J$. For $3 d$ transition metal elements in bulk materials the repulsion $U$ is around 2-3 eV and $J$ is around $0.9 \mathrm{eV}^{36}$. Due to the lower coordination of the Co atom on the graphene sheet the screening should be reduced compared to its bulk value. Here we take $U=5 \mathrm{eV}$ and $J=0.9 \mathrm{eV}$ as in our previous work ${ }^{31}$.

The Coulomb interaction within the correlated $3 d$ subspace has already been taken into account on a static mean-field level in the effective KS Hamiltonian of the central unit cell C. Therefore the KS Hamiltonian within the correlated subspace $\mathbf{h}_{3 d}^{\mathrm{KS}}$ has to be corrected by a double-counting correction term, i.e. $\mathbf{h}_{3 d} \equiv \mathbf{h}_{3 d}^{\mathrm{KS}}-\mathbf{h}_{\mathrm{dcc}}$. Here we use the standard expression,

$$
\mathbf{h}_{\mathrm{dcc}}=\left[U\left(N_{3 d}-1 / 2\right)-J\left(N_{3 d}-1\right) / 2\right] \times \mathbf{1}_{3 d}
$$

where $\mathbf{1}_{3 d}$ is the identity matrix in the Co $3 d$-subspace, and $N_{3 d}$ is the occupation of the impurity $3 d$-shell ${ }^{37}$.

In order to compute the electronic self-energy $\Sigma_{3 d}$ we have to solve the generalized Anderson impurity problem given by the strongly interacting Co $3 d$-electrons. The impurity problem is completely determined by the interacting Hamiltonian of the Co $3 d$-shell, i.e. $\hat{\mathcal{H}}_{3 d}=\hat{h}_{3 d}+$ $\hat{\mathcal{H}}_{U}$, and by the so-called hybridization function $\boldsymbol{\Delta}_{3 d}(\omega)$ which describes the dynamic hybridization of the Co $3 d-$ shell with the rest of the system (i.e. the bath). The hybridization function can be extracted from the noninteracting Green's function $\left[\mathbf{G}_{\mathrm{C}}^{0}\right]^{-1} \equiv\left[\mathbf{G}_{\mathrm{C}}\right]^{-1}+\boldsymbol{\Sigma}_{3 d}(\omega)$ of the central region $\mathrm{C}$ as follows:

$$
\boldsymbol{\Delta}_{3 d}(\omega)=\omega+\mu-\mathbf{h}_{3 d}-\left[\mathbf{g}_{3 d}^{0}(\omega)\right]^{-1}
$$

$\mathbf{g}_{3 d}^{0}$ is the non-interacting Green's function projected onto the $3 d$-shell of the Co atom, i.e. $\mathbf{g}_{3 d}^{0} \equiv \mathbf{P}_{3 d} \mathbf{G}_{\mathrm{C}}^{0} \mathbf{P}_{3 d}$. 
Solving the generalized Anderson impurity problem is a difficult task, and at present there is no universal impurity solver that works efficiently and accurately in all parameter regimes. Here we make use of impurity solvers based on an expansion in the hybridization strength given by $\Delta_{3 d}(\omega)$ around the atomic limit. The starting point is an exact diagonalization of the (isolated) impurity subspace i.e. the Co $3 d$-shell in our case given by the interacting Hamiltonian $\hat{\mathcal{H}}_{3 d}$. The hybridization of the impurity subspace with the rest of the system (given by the hybridization function $\left.\Delta_{3 d}(\omega)\right)$ is then treated perturbatively.

The so-called Non-Crossing Approximation (NCA) is a self-consistent perturbation expansion to lowest order in the hybridization strength. NCA only takes into account the most simple diagrams describing simple hopping processes where an electron or hole hops into the impurity at some time and then out at a later time (see Fig. 7 in App. B). The so-called One-Crossing Approximation (OCA) improves upon the NCA by taking into account second order diagrams where two additional electrons (holes) are acommodated on the impurity at the same time in addition to the NCA diagrams as shown in Fig. 7, OCA is the lowest order self-consistent approximation that is exact up to first order in the hybridization $\Delta_{3 d} \propto V^{2}$ for all physical quantities while NCA is only exact to first order in $\Delta_{3 d}$ for the conduction electron self-energy. OCA improves considerably many of the shortcomings of NCA: It substantially improves the width of the Kondo peak and hence the Kondo temperature which now are only slightly underestimated. It also corrects the asymmetry of the Kondo peak. For very low temperatures ( $T \ll T_{K}$ ), however, the height of the Kondo peak is still overestimated, and the Fermi liquid behaviour at zero temperature is not recovered.

Hence, OCA is a reasonable approximation for solving the generalized impurity problem as long as the temperatures are not too low (i.e. more than one order of magnitude below $T_{K}$ ). In App. B we give a brief introduction to the NCA and OCA impurity solvers. A detailed description of the NCA and OCA methods can be found e.g. in Refs. 8,33 35.

\section{DFT ATOMIC AND ELECTRONIC STRUCTURE CALCULATIONS}

First, we have calculated the adsorption curves for a Co atom on a Graphene sheet with density-functional calculations within the generalized gradient approximation (GGA) with the CRYSTAL06 32 ab initio electronic structure program for periodic systems employing an elaborate all-electron Gaussian basis set (6-31g).

As possible adsorption sites for the Co impurity we consider the three high symmetry sites shown on the inset of the top panel of Fig. 1, i.e. the hollow, top, and bridge site. For the calculations we have chosen a $5 \times 5$ unit cell in order to avoid interactions between impurities in different
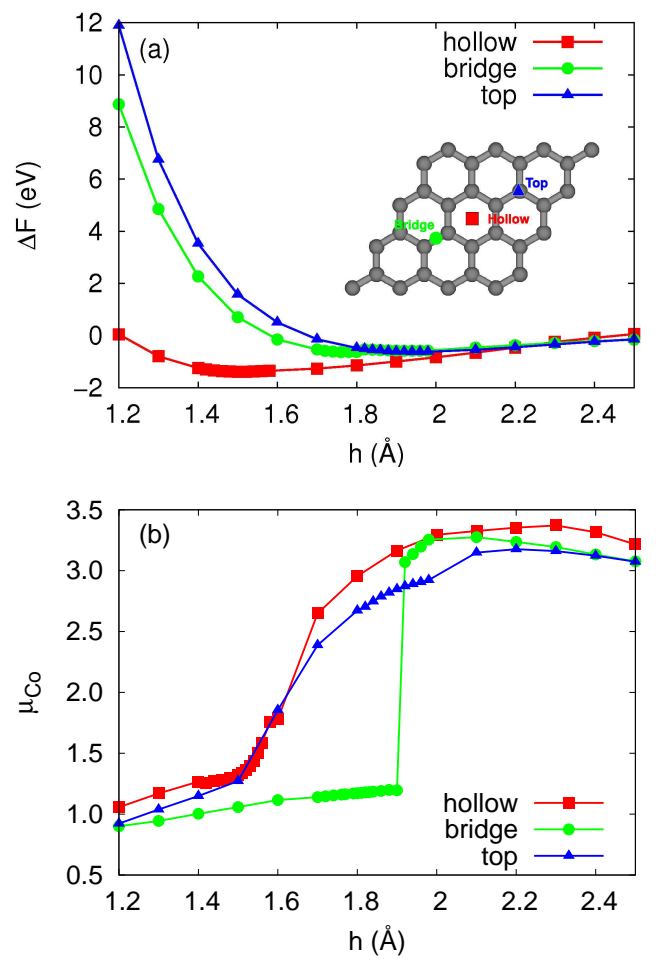

FIG. 1: (color online) Adsorption of Co atoms on graphene. (a) Adsorption energy of Co adatom on graphene sheet in dependence of distance $h$ for the three different adsorption sites as shown in the inset: Hollow (h), top (t), and bridge site (b). (b) Magnetic moment of Co adatom on graphene sheet in dependence of distance $h$ between Co atom and graphene sheet.

unit cells. We have checked that the results essentially do not change when choosing a slightly smaller $(4 \times 4)$ or bigger (6x6) unit cell. Fig. 1(a) shows the adsorption energy for a Co atom in dependence of the distance $h$ between the Co atom from the graphene sheet for the three different sites. One can see that the hollow site is the most favourable absorption site. We find the energy minimum for this site at a distance of $h=1.51 \AA$. As can be seen the energy minima for the other two adsorption sites are much more shallow. We find $h=1.81 \AA$ for the bridge site, and $h=1.96 \AA$ for the top site. The top site is somewhat more favourable in energy than the bridge site.

In Fig. 1(b) the magnetic moment of the Co atom in dependence of the height $h$ of the Co atom over the graphene sheet is shown. Initially the magnetic moment is 3, i.e. that of a free Co atom. When the Co atom approaches the equilibrium distance for a certain adsorption site the magnetic moment starts to decrease. At the equilibrium distance, the magnetic moment of the Co atom has decreased to below 1.5 for the hollow site while for the bridge site it is more close to 1 already. For the top site, on the other hand, the magnetic moment is still around 3 at the equilibrium distance as for the free atom. The magnetic moment for all three ad- 


\begin{tabular}{c|c|c|c|c|c|c|c} 
& $\mathrm{h}(\AA)$ & $E_{\mathrm{ad}}(\mathrm{eV})$ & $\Delta Q_{\mathrm{Co}}(e)$ & $\mu_{\mathrm{Co}}$ & $\mu$ & $N_{3 d}$ & $\mu_{3 d}$ \\
\hline $\mathrm{h}$ & 1.51 & -1.395 & 0.536 & 1.336 & 1.127 & 7.530 & 1.197 \\
$\mathrm{~b}$ & 1.81 & -0.660 & 0.354 & 1.175 & 1.109 & 7.533 & 1.627 \\
$\mathrm{t}$ & 1.96 & -0.628 & 0.248 & 2.905 & 2.913 & 7.365 & 2.150 \\
$\mathrm{~h}^{*}$ & 1.87 & - & 0.311 & 3.065 & 2.890 & 7.337 & 2.150
\end{tabular}

TABLE I: Summary of GGA results. Optimal height $(h)$ of Co atom, adsorption energy $\left(E_{\mathrm{ad}}\right)$, charge $\left(\Delta Q_{\mathrm{Co}}\right)$ and magnetic moment $\left(\mu_{\mathrm{Co}}\right)$ of Co atom, total magnetic moment of unit cell $(\mu)$, occupation $\left(N_{3 d}\right)$ and magnetic moment $\mu_{3 d}$ of Co $3 d$-shell for the different adsorption sites considered here, i.e. $\mathrm{h}$ (hollow), b (bridge), t (top). In the last row $\left(\mathrm{h}^{*}\right)$, we show the GGA results for the Co atom at the hollow site for the optimal distance $h=1.87 \AA$ calculated with the B3LYP hybrid functional (see text).

sorption sites approaches 1 when further decreasing the height of the Co atom. In the case of the bridge adsorption site the transition from the free magnetic moment of $3 \mu_{B}$ to a magnetic moment of $1 \mu_{B}$ is remarkably abrupt. A summary of our GGA results is given in Tab. I. Our GGA results are in good agreement with previous work by other groups using different methods 24.39 . In particular, we find for the hollow adsorption site at the equilibrium distance that the magnetic moment of the $3 d$-shell is indeed close enough to 1, implying a spin$1 / 2$ for the Co $3 d$-shell, as reported by Wehling et al 24 . However, the occupation of the $3 d$-shell is around 7.5 and not around 9 as reported by Wehling et al. As will be explained below the approximate spin- $1 / 2$ of the Co $3 d$ is essentially due to a hole in the $\mathrm{E}_{1}$ minority-spin levels.

We have also performed hybrid functional calculations ${ }^{41}$ which in general yield better geometries for molecules. Using the GAUSSIAN09 $\stackrel{40}{\underline{4}}$ quantum chemistry code we have opimized the distance $h$ of the Co atom on the hollow site of a finite graphene flake of hexagonal shape with $24 \mathrm{C}$ atoms and with $\mathrm{H}$ atoms at the borders to saturate the bonds $\frac{42}{2}$. Employing the popular B3LYP hybrid functional together with the 6-31G basis set (also employed before in the CRYSTAL06 calculations) we now find a considerably higher value for the optimal height of the Co atom at the hollow site, namely $h=1.87 \AA$. For the GGA functionial we obtain very similar results $(h=1.47 \AA)$ as before with CRYSTAL06 code. At the optimal height calculated with B3LYP ( $h=1.87 \AA)$ both, the GGA and the B3LYP results are remarkably similar with respect to the magnetic moments and occupations of the Co $3 d$-shell: The occupation of the $3 d$-shell is 7.4 and the corresponding magnetic moment is close to 2 now, implying a total spin of 1 for the $3 d$-shell. In the last row of Tab. II we have added the GGA results for the hollow site with the Co atom at the optimal distance $h=1.87 \AA$ calculated with the B3LYP functional.

In Fig. 2, we show the Co $3 d$-spectral functions calculated with spin-polarized GGA for the Co atom at the hollow site for two different heights, i.e. the optimal height predicted by GGA ( $h=1.51 \AA$ ) and the optimal
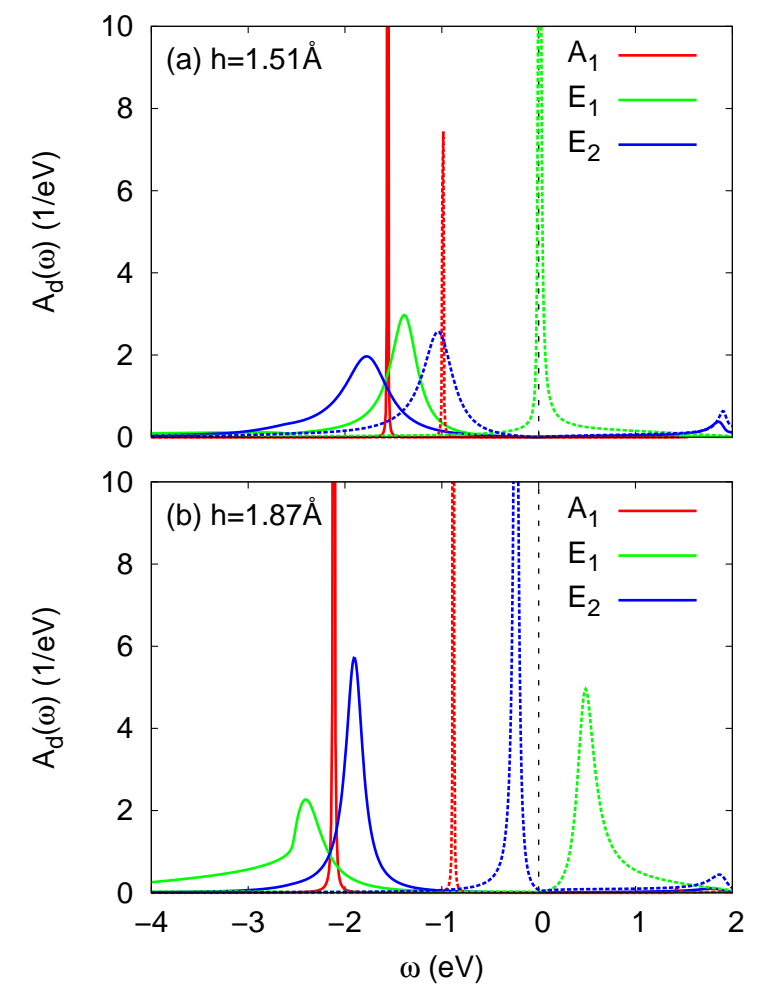

FIG. 2: (color online) Co $3 d$-spectral densities calculated with spin-polarized GGA for Co atom at hollow site for (a) the GGA equilibrium distance $h=1.51 \AA$ and (b) the B3LYP equlibrium distance $h^{*}=1.87 \AA$. Full (dashed) lines correspond to spin-up (down) states. The vertical dashed line indicates the Fermi level.

height ( $h=1.87 \AA$ ) predicted by B3LYP. Due to the C6symmetry of the system the Co $3 d$-shell splits into three symmetry groups: The non-degenerate $\mathrm{A}_{1}$ group consist-

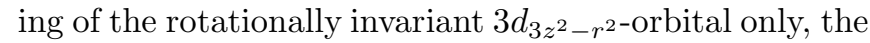
doubly degenerate $\mathrm{E}_{1}$ group consisting of the $3 d_{x z^{-}}$and $3 d_{y z}$-orbitals and the $\mathrm{E}_{2}$ group consisting of the $3 d_{x y}$-and

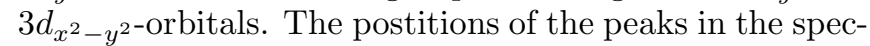
tral functions show the effective energy levels for each of the symmetry groups. We can see that at the GGA equilibrium height (Fig. 2(a)) the spin- $1 / 2$ is essentially due to a hole in the $\mathrm{E}_{1}$-levels. The $\mathrm{E}_{1}$ minority-spin levels are exactly at the Fermi level and hence are half-filled, while the $\mathrm{E}_{1}$ majority-spin and the $\mathrm{A}_{1}$ and $\mathrm{E}_{2}$ minorityand majority-spin levels are well below the Fermi level. This level scheme seems to suggest a $3 d^{9}$ configuration as reported by Wehling et al $\stackrel{24}{4}$, although the true occupation of the $3 d$-shell is really only 7.5. The reason for this apparant discrepancy is the dynamic hybridization of the Co $3 d$-levels with the graphene substrate.

The Co $3 d$-spectral density calculated at the B3LYP equilibrium distance is shown in Fig. 2(b). The main difference with the spectral density at the GGA equilibrium (Fig. 2(a)) is that the peak corresponding to the minority-spin $\mathrm{E}_{1}$-levels are now completely above the 


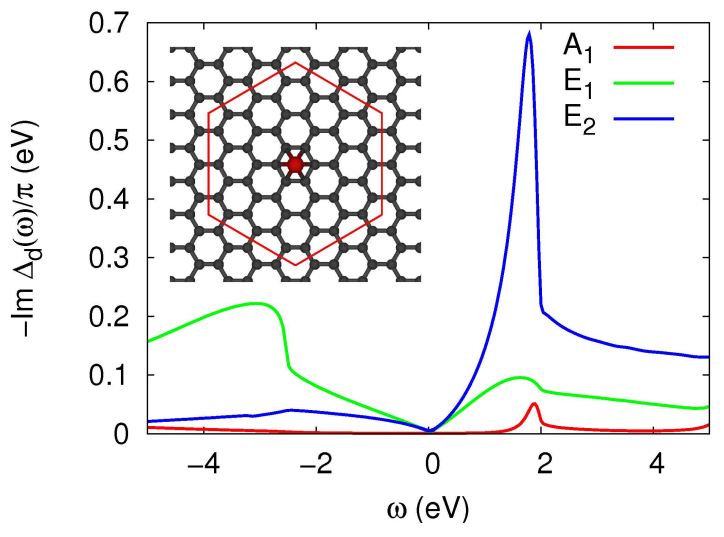

FIG. 3: (color online) Imaginary part of the hybridization functions for the Co atom at the hollow site as shown in the inset for $h=1.87 \AA$ and for the three symmetry groups. The inset shows a graphene sheet with Co adatom at hollow site The red hexagon indicates the central region $\mathrm{C}$ containing 54 carbon atoms.

Fermi level, and hence the minority-spin $\mathrm{E}_{1}$-levels are completely empty. Hence the $3 d$-shell now yields a magnetic moment of about 2, i.e. a spin- 1 due to two holes in the $\mathrm{E}_{1}$-levels. Note that now the total magnetic moment of the Co atom is 3 due to a single electron in the Co $4 s$-orbital which is fully spin-polarized. Also note that in this geometry, both the $\mathrm{A}_{1}$ - and the $4 s$-level do not couple at all to the graphene substrate for symmetry reasons 24 . Our GGA results at the B3LYP equilibrium distance show some similarity to the GGA+U results of Wehling et al ${ }^{25}$

\section{GGA+OCA CALCULATIONS FOR CO ATOM ON HOLLOW SITE}

The inset of Fig. 3 shows the central region $\mathrm{C}$ containing the Co adatom at the hollow site embeded into a perfect graphene sheet. The cell has been taken large enough in order to avoid interaction between Co atoms in the CRYSTAL periodic supercell calculation. The shape of the unit cell has been chosen to reflect the 6-fold symmetry of the system. The $\mathrm{C}$ region can now be embeded directly into the graphene lattice set up by supercells of the same shape as C as described before in Sec. III.

Due to the C6-symmetry of the system the Co $3 d-$ shell splits into three symmetry groups: The nondegenerate $A_{1}$ group consisting of the rotationally in-

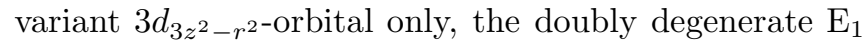
group consisting of the $3 d_{x z^{-}}$and $3 d_{y z}$-orbitals and the $\mathrm{E}_{2}$ group consisting of the $3 d_{x y^{-}}$and $3 d_{x^{2}-y^{2}}$-orbitals.

The right panel of Fig. 3 shows the hybridization functions for each symmetry group. As can be seen at low

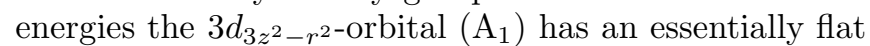
and zero hybridization with the graphene sheet. This

\begin{tabular}{c|c|c|c} 
& $\epsilon_{3 d}^{\mathrm{KS}}(\mathrm{eV})$ & $\operatorname{Re} \Delta_{3 d}(\mathrm{eV})$ & $\tilde{\epsilon}_{3 d}^{\mathrm{KS}}(\mathrm{eV})$ \\
\hline $\mathrm{A}_{1}$ & -3.650 & -0.144 & -3.794 \\
$\mathrm{E}_{1}$ & -3.637 & +0.197 & -3.440 \\
$\mathrm{E}_{2}$ & -3.414 & -0.389 & -3.803
\end{tabular}

TABLE II: Crystal field splittings for $3 d$-shell of Co atom on hollow site of graphene $(h=1.87 \AA) . \epsilon_{3 d}^{\mathrm{KS}}$ are the onsite energies before double-counting correction, $\operatorname{Re} \Delta_{3 d}$ is the real part of the hybridization function at the Fermi level, and $\tilde{\epsilon}_{3 d}^{\mathrm{KS}} \equiv \epsilon_{3 d}^{\mathrm{KS}}+\operatorname{Re} \Delta_{3 d}(0)$ are the effective on-site energies taking into account the real part of the hybridization function. Note that the $A_{1}$ and $E_{2}$ effective energy levels are almost degenerate.

can be understood by the fact that the coupling of the $3 d_{3 z^{2}-r^{2}}$ on the hollow site to the $2 p_{z}$-orbitals of the graphene sheet is zero due to symmetry considerations ${ }^{24}$. The $\mathrm{E}_{2}$-group has the (overall) strongest coupling to the C $2 p_{z}$-orbitals in that geometry as can be seen from the corresponding hybridization function. Note that the hybridization function for both the $\mathrm{E}_{1}$ and $\mathrm{E}_{2}$ symmetry group vanish linearly near the Fermi energy reflecting the linear DOS of the graphene sheet. Interestingly, the slopes for the $\mathrm{E}_{1}$ - and $\mathrm{E}_{2}$-hybridization functions are different for energies below and above the Fermi level. Morover, for negative energies the $\mathrm{E}_{1}$-hybridization function has a steeper slope than the $\mathrm{E}_{2}$-hybridization, while for positive energies it is the other way round. This implies that $\mathrm{E}_{1}$ couples predominantly to the negative energy band of graphene while $E_{2}$ couples predominantly to the positive energy band.

In Table. III we show the energy levels, the real part of the hybridization function at the Fermi level, and the effective energy levels of the Co $3 d$-shell. While the splitting of the bare energy levels $\epsilon_{3 d}$ is around $0.2 \mathrm{eV}$, the splitting of the effective energy levels $\tilde{\epsilon}_{3 d}$ taking into account the (real part of the) hybridization with the graphene is somewhat bigger with around $0.4 \mathrm{eV}$. Taking into account the real part of the hybridization changes also the ordering of the energy levels. For the ordering of the effective energy levels we obtain: $\tilde{\epsilon}\left(E_{2}\right)<\tilde{\epsilon}\left(A_{1}\right)<$ $\tilde{\epsilon}\left(E_{1}\right)$. Note that this level ordering is similar to the one obtained by Wehling et $a l{ }^{25}$.

\section{A. GGA+OCA results}

We now solve the generalized impurity problem of the Co $3 d$-shell coupled to the graphene sheet by using the OCA impurity solver as described in Sec. III and in App. B. We take the Coulomb repulsion $U$ between $d$-orbitals to be somewhat higher than in bulk due to the supposedly smaller screening: $U=5 \mathrm{eV}$. The Hund's rule coupling is taken to be the same as in bulk, i.e. $J=0.9 \mathrm{eV}$ since it is less affected by screening effects. The DCC was calculated by the standard expression given by eq. (31) and taking the GGA value of about 7.5 for $N_{3 d}$. With OCA, the total occupation of the impurity $3 d$-shell is 

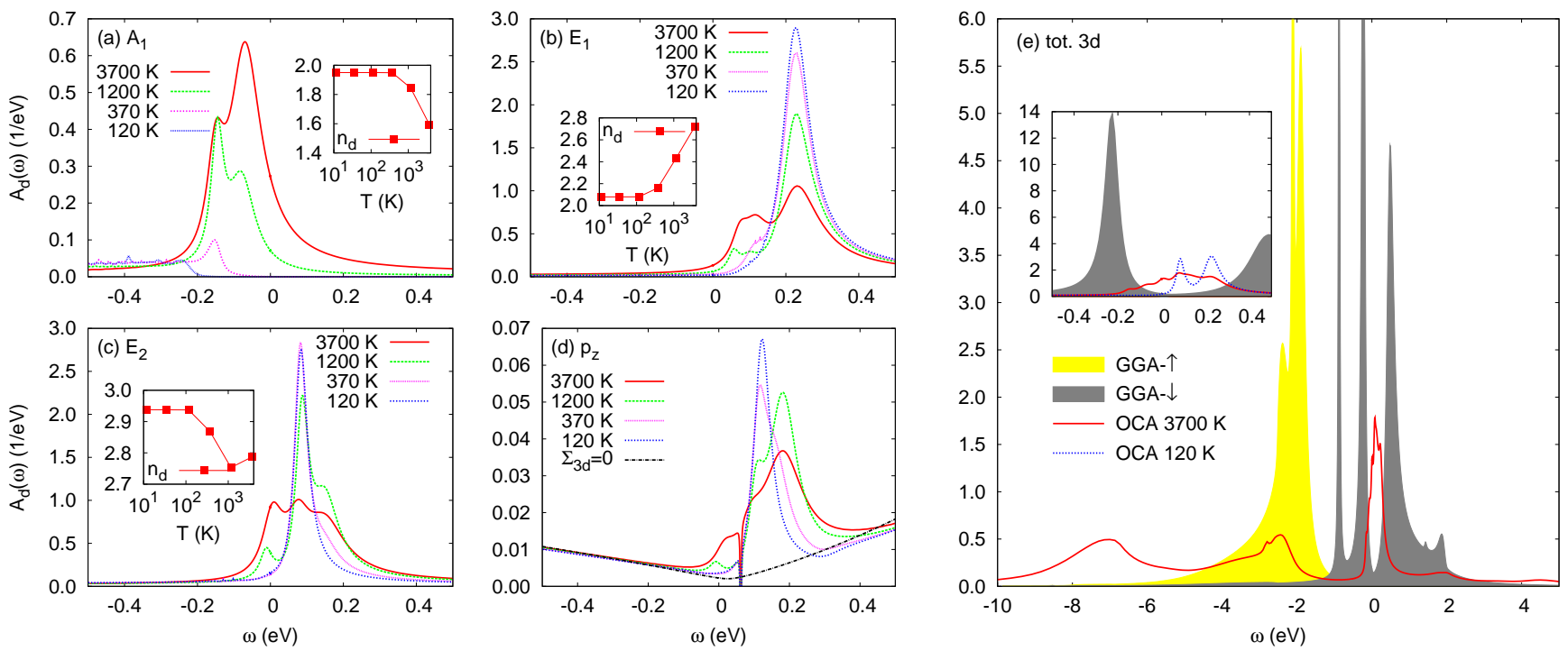

FIG. 4: (color online) GGA+OCA results for $U=5 \mathrm{eV}$ and $J=0.9 \mathrm{eV}$ and total occupation $N_{3 d}$ of the $3 d$-shell around 7.0 . (a-c) Co $3 d$-spectral functions near the Fermi level for all three symmetry groups at different temperatures. The insets show the corresponding orbital occupations as a function of temperature. (d) Spectral function of $p_{z}$-orbital of carbon atoms near the Co-atom for different temperatures. The black dashed line shows the $p_{z}$-spectral function when the self-energy of the Co $3 d$-electrons $\Sigma_{3 d}$ is set to zero. (e) Comparison of GGA+OCA $3 d$-spectral density with spin-polarized GGA spectral density on a large energy scale. The inset shows a blow-up of the region near the Fermi level.

now between 7.1 and 7.0 at low temperatures compared to the GGA value of 7.5. This result is very typical for correlated calculations: The correlations push the system towards integer occupation numbers to lower the energy.

Fig. [4 shows the spectral functions of the Co $3 d$-electrons for different temperatures calculated with OCA. At high temperatures we find peaks near the Fermi level for all three symmetry groups. However, the temperature dependencies of the individual peaks are quite different from each other: For the $\mathrm{A}_{1}$-symmetry consisting of the $d_{3 z^{2}-r^{2} \text {-orbital the peak becomes smaller at }}$ increasing temperatures and finally develops a step-like behaviour at low temperatures. The peaks in the $\mathrm{E}_{1}$ and $\mathrm{E}_{2}$-spectra on the other hand become more pronounced with decreasing temperature more or less as expected for Kondo effect.

The form of the spectral functions and the temperature behaviour can be understood by looking at the occupation numbers for the different symmetry groups. The insets of Figs. 4(a-c) show the occupation numbers as a function of temperature for each of the three symmetry groups. For the $\mathrm{A}_{1}$-symmetry the occupation changes from $\approx 1.6$ at high temperatures to almost 2 at low temperatures. Hence, at low temperatures the

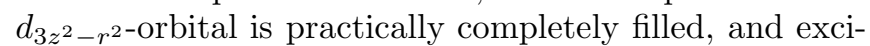
tations near the Fermi level are suppressed. For the $\mathrm{E}_{1}$ symmetry group composed of the $d_{x z^{-}}$and $d_{y z^{-}}$orbitals the occupation changes from 2.8 at high temperatures to 2.1 at low temperatures. Hence at low temperatures this doubly-degenerate channel is essentially half-filled, corresponding to a spin of almost 1 in this channel due to Hund's rule coupling. The peak near the Fermi level in the $\mathrm{E}_{1}$-spectral function is hence due to a spin- 1 Kondo effect in this channel. For the $\mathrm{E}_{2}$-symmetry composed of the $d_{x y^{-}}$and $d_{x^{2}-y^{2}}$-orbitals we find an occupation of 2.8 for high temperatures, and of almost 3 for low temperatures. Hence the doubly-degenerate $\mathrm{E}_{2}$-channel accomodates 3 electrons with a total spin- $1 / 2$ so that the peak in the $\mathrm{E}_{2}$-spectral function is due to a spin$1 / 2$ Kondo effect. Accordingly, the peaks near the Fermi level in the $3 d$-spectral function at low temperature are due to a spin- $3 / 2$ Kondo effect in the $3 d$-levels. This interpretation is further corroborated by the fact that the main contribution to the electronic configuration of the $3 d$-shell is an eightfold degenerate state with $N_{d}=7$ and $S=3 / 2$. The very fact that such a high spin state can be screened by the graphene substrate is an evidence of the multi-channel character of the graphene as a conduction electron bath.

In Fig. 4(e) we compare the $3 d$-spectral density calculated with the GGA+OCA (at $T=120 \mathrm{~K}$ ) method on the one hand and with spin-polarized GGA on the other hand. As can be seen the spectral densities calculated by the two methods differ significantly from each other. In the GGA spectrum, the peak just below the Fermi level (see also the inset of Fig. 4(e)) corresponds to a completely filled $E_{1}$-shell with down-spin as can be seen by comparison with Fig. 3(c). The peak above (and also a little farther from) the Fermi level corresponds to a completely empty spin-down $E_{2}$-shell. Both peaks carry essentially the full spectral weight of the correpsonding orbitals. In contrast, the two peaks near the Fermi level in the GGA+OCA spectra which are also of $E_{1^{-}}$and $E_{2^{-}}$ symmetry, do not carry the full spectral weight of the 
corresponding orbitals due to the renormalization of the quasi-particle by the electron-electron interactions which cannot be captured by a static mean-field calculation like GGA.

Fig. 4(d) shows that the correlations in the Co $3 d$ shell also affect the projected DOS of the $p_{z}$-orbitals on the carbon atoms that are directly coupled to the Co atom (i.e. the 6 carbon atoms nearest to the Co atom). This can be understood as follows: Due to the coupling between the carbon $p_{z^{-}}$and the Co $3 d$-orbitals, the full $p_{Z}$-Green's function $G_{p}(\omega)$ is related to the full Green's of the $3 d$-orbitals $G_{d}(\omega)$ by:

$$
G_{p}(\omega)=G_{p}^{0}(\omega)+\sum_{d} G_{p}^{0}(\omega) V_{p d} G_{d}(\omega) V_{d p} G_{p}^{0}(\omega)
$$

where $G_{p}^{0}(\omega)$ is the Green's function of the (unperturbed) graphene $p_{z}$-orbitals without the coupling to the Co $3 d$ orbitals and $V_{p d}=V_{d p}^{*}$ is the coupling between the $p_{z^{-}}$ and individual $3 d$-orbitals. The full $3 d$-Green's function $G_{d}(\omega)$ takes into account the correlations given by $\Sigma_{3 d}(\omega)$ and the hybridization with the $p_{z}$-orbitals given by $\Delta_{3 d}(\omega)$, i.e. $V_{p d} G_{d}(\omega) V_{d p}$ represents the full $T$-matrix for the $p_{z}$-electrons.

One can see a temperature-dependent peak roughly at the position of the peaks in the $E_{1^{-}}$and $E_{2}$-spectral density. For comparison we also show the $p_{z}$-DOS when the self-energy of the Co $3 d$-electrons $\Sigma_{3 d}$ is set to zero. In this case the $3 d$-levels are far below the Fermi level and hence cannot affect the $p_{z}$-DOS near the Fermi level. Hence the sharp resonance in the $p_{z}$-DOS vanishes. This shows that the Kondo peaks in the Co $3 d$-spectra can also be detected indirectly by spectroscopy of the nearby carbon atoms.

\section{B. Dependence on $3 d$-energy levels}

The double-counting correction term $\mathbf{h}_{\mathrm{dcc}}$ given by eq. (3) which substracts out the Coulomb interaction within the correlated $3 d$-subspace that has already been taken into account on a static mean-field level in the LDA or GGA calculation is not known exactly. Hence the exact position of the $3 d$-energy levels is not known. Here we explore how shifting the $3 d$-energy levels changes the results.

Figs. 5(a-c) show the Co $3 d$-spectral densities for each of the different symmetries and for different shifts $\Delta \epsilon_{d}$ of the $3 d$-levels with respect to their original position, and Figs. 5],e the corresponding occupancies. Quite obviously there is a drastic change in the correlations when the impurity-levels are lowered by more than $0.2 \mathrm{eV}$. At that point, a substantial peak appears in the $\mathrm{A}_{1}$-spectra, while the peak in the $\mathrm{E}_{1}$-spectra disappears. The peak in the $\mathrm{E}_{2}$-spectra on the other hand becomes qualitatively different for energy shifts $\Delta \epsilon_{d} \leq-0.2 \mathrm{eV}$, i.e. their spectral weight increases considerably. The changes in the spectra are accompanied by changes in the occupancies: Most importantly, the occupancy of the $\mathrm{E}_{2}$-levels
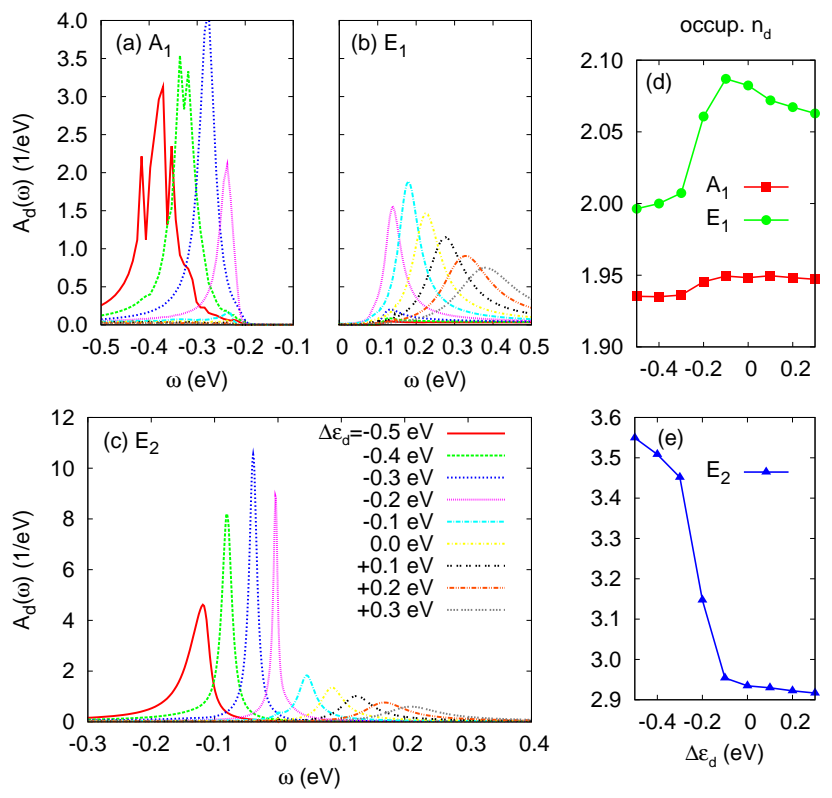

FIG. 5: (color online) 3d-energy level dependence. (a-c) Spectral functions of Co $3 d$-levels for different energy shifts $\Delta \epsilon_{d}$ of the $3 d$-levels (as indicated in the legend of (c)) at $T=37 \mathrm{~K}$ and for the three different symmetries. (d,e) Orbital occupancies of the Co $3 d$-levels as a function of the energy shift for each of the three symmetries and for $T=37 \mathrm{~K}$.

increases abruptly by about 0.6 for $\Delta \epsilon_{d} \approx-0.2 \mathrm{eV}$. The changes in the $3 d$-levels with $\mathrm{A}_{1}$ - and $\mathrm{E}_{1}$-symmetry are less pronounced but also occur at the same point. Also the overall occupation of the impurity levels changes quite abruptly by about 0.5 electrons from $N_{d} \approx 7$ to $N_{d} \approx 7.5$. This indicates a strong change in the electronic configuration of the Co $3 d$-shell.

Indeed we find that for an energy shift around $-0.2 \mathrm{eV}$, another $3 d$-shell state with $N_{d}=8$ and $S=1$ becomes similar in energy to the state with $N_{d}=7$ and $S=3 / 2$. Hence, at this point the system enters the mixed-valence regime. This explains the strong increase of the spectral weight in the $\mathrm{E}_{2}$-peak for $\Delta \epsilon_{d} \leq-0.2 \mathrm{eV}$ since the mixedvalence regime is characterized by a strong peak near the Fermi level. On the other hand, the integer occupation of 2 in the $E_{1}$-levels, and the associated spin-1, essentially does not change upon entering the mixed-valence regime. In fact, the occupation is now even closer to 2 than it was in the Kondo regime. Also the occupation of the $\mathrm{A}_{1}$-level remains essentially constant upon entering the mixed-valence regime. Hence the mixed-valence is actually only induced in the $\mathrm{E}_{2}$-levels since the occupations of the $\mathrm{A}_{1}$-level and the $\mathrm{E}_{1}$-levels in the $N_{d}=8$ state are the same as in the $N_{d}=7$ state. The vanishing of the peak in the $\mathrm{E}_{1}$-spectra upon entering the mixedvalence regime is due to the weaker hybridization with the graphene for negative energies (compare Fig. 31): As the Kondo peak in the $\mathrm{E}_{1}$-levels moves beyond the Dirac point as the impurity-levels are lowered in energy, the Kondo coupling in the $\mathrm{E}_{1}$-levels becomes weaker and the 
Kondo temperature much smaller so that the Kondo effect is not observed anymore at the temperatures considered here. It is not quite clear what triggers the peak in the $A_{1}$-spectra upon entering the mixed-valence regime. One possibility is that the increased charge fluctuations in the mixed valence regime also lead to charge fluctuations in this level via the Hund's rule coupling to the other $3 d$-levels. The slight decrease in the occupation of the $\mathrm{A}_{1}$-level in the mixed-valence regime supports this view.

Note that the position of a peak (if present) changes according to the energy shift. This also explains the change in the width of the peaks for the $\mathrm{E}_{1}$ - and $\mathrm{E}_{2}$ - symmetries: As a peak moves away from the Fermi level the broadening increases due to the increased hybridization with the graphene substrate. The $\mathrm{A}_{1}$-level on the other hand does not couple at all to the graphene substrate (zero hybridization) so that the changes in the shape of the $\mathrm{A}_{1}$-peak are purely correlation effects.

Hence tuning the energies of the Co $3 d$-levels changes the correlations in these levels due to changes in the occupancy. More precisely, the system is driven from the Kondo regime to the mixed-valence regime when the energies of the $3 d$-levels are lowered by more than $0.2 \mathrm{eV}$ with respect to their original energies. The change in the correlations is reflected by corresponding changes in the $3 d$-spectral functions.

\section{Gate voltage dependence}

In the previous subsection we have seen that changing the exact position of the Co $3 d$-levels with respect to the Fermi level has a considerable effect on the electronic correlations and hence on the spectral density of the $3 d$-levels. Although changing the impurity levels directly is not feasible in an experiment, one can instead easily tune the chemical potential of graphene by application of a gate voltage. The chemical potential then is shifted with respect to both, the impurity levels and the graphene bands. Hence it should be possible to control the electronic correlations and in particular the Kondo effect by application of a gate voltage. The change in correlations can then be detected in the $3 d$-spectra for example by STM spectroscopy.

More precisely, a gate voltage $v_{g}$ shifts the chemical potential $\mu$ with respect to the graphene bands and the impurity levels according to $\mu=\epsilon_{F}-e v_{g}$ where $e$ denotes the elementary charge and $\epsilon_{F}$ is the Fermi level of neutral graphene, i.e. the energy of the Dirac points (see inset of Fig. 6a). Overall the results for shifting the chemical potential presented in Fig. 6 look very similar to the previous ones for shifting the impurity levels in Fig. 5 .

At the hollow site only the Co $3 d$-levels of $\mathrm{E}_{1^{-}}$and $\mathrm{E}_{2^{-}}$ symmetry couple to the graphene sheet for low energies while the $\mathrm{A}_{1}$-level is completely decoupled. Hence only the spectra of $\mathrm{E}_{1}$ - and $\mathrm{E}_{2}$-levels can be measured by an STM. Therefore in Fig. 6(a), we show the total spectral density of the $\mathrm{Co}_{1}$ - and $\mathrm{E}_{2}$-levels for different values of the gate voltage. For gate voltages smaller than $-0.1 \mathrm{eV}$ there is only one peak in the spectral function which is due to the $\mathrm{E}_{2}$-levels. For gate voltages $\geq-0.1 \mathrm{eV}$ a second peak appears which originates from the $\mathrm{E}_{1}$-levels. The $E_{2}$-peak and also the $E_{1}$-peak (when present) move with respect to the chemical potential $\mu$ according to the change in gate voltage. Hence the peaks in the spectral function are not pinned to the chemical potential as expected for normal Kondo effect, but are rather pinned to the Dirac points of the graphene sheet. For completeness we also show the spectral density of the $A_{1}$-level in Fig. 6b for different gate voltages. A peak in the spectral density at low temperature is only observed for negative bias voltages. The peak disappears for zero and positive gate voltage. Also the resonance in the $\mathrm{A}_{1}$-level appears to be pinned to the Dirac point of graphene rather than to the chemical potential.

As can be seen in Figs. [6 d,e also the individual occupations of the $3 d$-levels as a function of the applied gate voltage show a similar behaviour as before as a function of the energy shift $\Delta \epsilon_{d}$ of the $3 d$-levels. In fact, the explanation for the behaviour of the spectra and occupancies as a function of the energy shift of the $3 d$-levels also applies to their behaviour as a function of the gate voltage. Here, when the gate voltage becomes smaller than $-0.1 \mathrm{~V}$ the system leaves the Kondo regime with an $N_{d}=7$ and $S=3 / 2$ state as the principal contribution to the electronic configuration of the $3 d$-shell, and enters the mixed-valence regime with an equal contribution coming from an $N_{d}=8$ and $S=1$ state.

We can estimate a Kondo temperature from the width of the resonance in the Kondo regime. Fig. [6] shows the Kondo temperature of the $\mathrm{E}_{2}$-levels estimated from the resonance width $\Gamma$ using the relation $k_{B} T_{K}=\pi \cdot w \cdot \Gamma / 8$ known from the Fermi liquid theory of the Anderson model where $w$ is Wilson's number 0.4128 (see e.g. the book by Hewson ${ }^{8}$, chap. 5). In Fig. 66 we have also included "Kondo temperatures" estimated from the width of the peaks for negative gate voltages where the system is not in the Kondo regime anymore. Hence outside the Kondo regime it is only a measure for the width of the resonance near the chemical potential.

Note that in the Kondo regime (i.e. for $v_{g} \geq 0$ ) the width of the resonance and hence the Kondo temperature are essentially constant. This behaviour is different from the one before where we only had shifted the impurity levels. There the width of the $\mathrm{E}_{1}$ - and $\mathrm{E}_{2}$-peaks increased as they moved away from the Fermi level due to the linear increase of the hybridization with the graphene substrate. Here, however, the peak is pinned to the Dirac point of graphene, i.e. it moves together with the graphene bands with respect to the chemical potential. Hence the hybridization with the graphene conduction electrons does not change here. The strong dependence of the width of the resonance on the gate voltage for negative gate voltages on the other hand is due to the changes in the electronic correlations as the system is driven out of the 

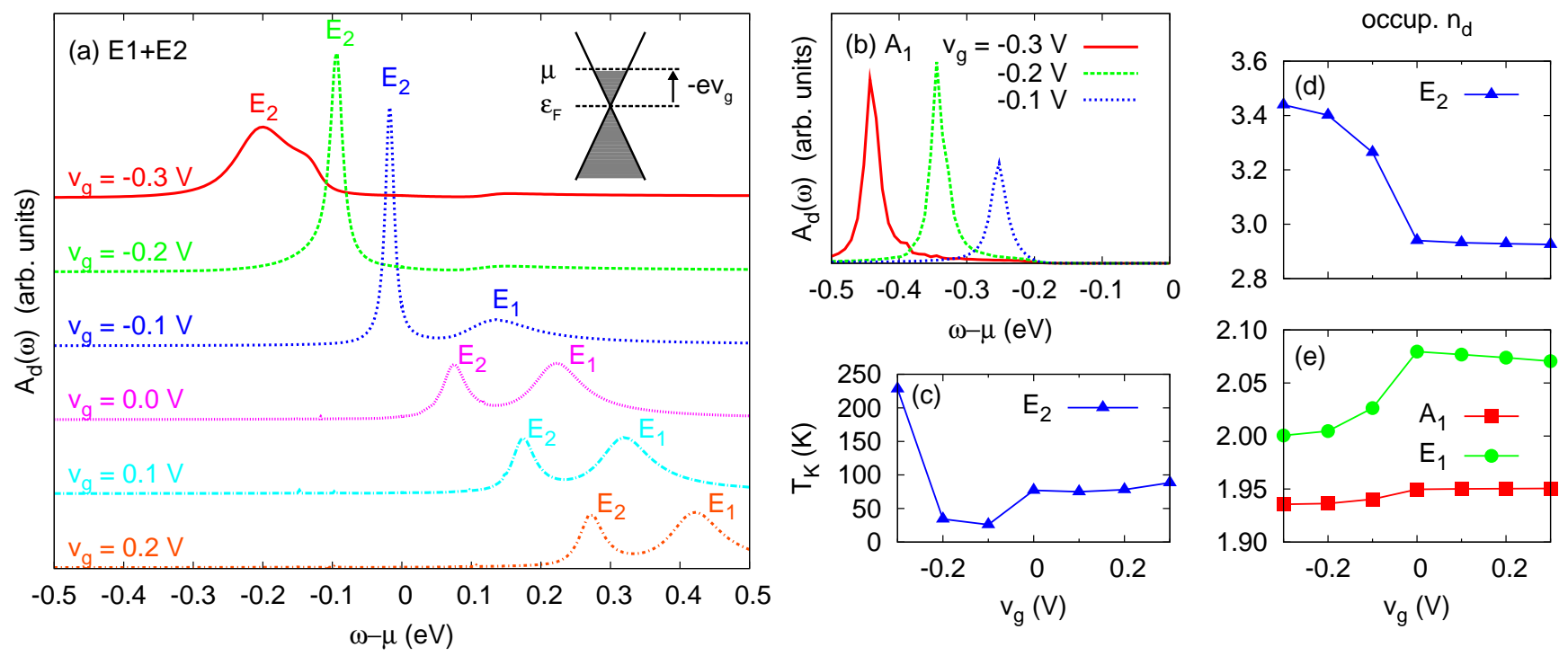

FIG. 6: (color online) Gate voltage dependence. (a) 3d-spectral function for $\mathrm{E}_{1}$ and $\mathrm{E}_{2}$ symmetry for different gate voltages $v_{g}(T=37 \mathrm{~K})$. Different offsets have been added to the spectral functions of different $v_{g}$ in order to better distinguish them from each other. The inset shows a Dirac cone of the graphene sheet with the position of the chemical potential $\mu$ for a certain gate voltage $v_{g}$. (b) Spectral function for Co $3 d$-level of $\mathrm{A}_{1}$-symmetry for different gate voltages $v_{g}(T=37 \mathrm{~K})$. The chemical potential in both (a) and (b) is always at zero frequency. (c) Kondo temperature estimated from width of $\mathrm{E}_{2}$ resonance as a function of gate voltage $v_{g}$ at $T=37 \mathrm{~K}$. (d-e) Occupancies of Co $3 d$-levels resolved for the three different symmetries as a function of gate voltage $v_{g}$ at $T=37 \mathrm{~K}$.

Kondo regime.

\section{SUMMARY AND CONCLUSIONS}

Using DFT electronic structure calculations we have found that a single Co atom most likely adsorbs at the hollow site of an otherwise clean graphene sheet. Furthermore the DFT calculations show, that the magnetic moment and the electronic configuration of the $3 d$-shell of the Co atom depends quite strongly on the adsorption height over the graphene sheet. At large distantes the formation of a local moment is favoured while at short distances the magnetic moment is quenched due to strong hybridization with the substrate. Since the adsorption energy curves are quite shallow, it might be possible in an experiment to push the Co atom from the local moment to the weakly correlated regime.

Using our GGA+OCA correlated electronic structure method for nanoscopic systems, we have then studied the impact of dynamical correlations due to the strongly interacting Co $3 d$-electrons on the electronic structure. Because of the strong crystal field splitting of the $3 d-$ levels by graphene substrate, the behaviour of each of the Co $3 d$-levels strongly depends on its orbital symmetry.

We find that the dynamic correlations can give rise to a spin- $3 / 2$ Kondo effect. The spin- $3 / 2$ is composed of a spin-1 due to two electrons within the doublydegenerate orbitals of $E_{1}$-symmetry and a spin- $1 / 2$ due to a hole within the doubly-degenerate orbitals of the $\mathrm{E}_{2}$ - symmetry. The Kondo effect gives rise to corresponding peaks near the Fermi level in the $\mathrm{E}_{1}$ - and $\mathrm{E}_{2}$-spectral function. The $\mathrm{A}_{1}$-channel on the other hand is nearly completely filled and hence in the Kondo regime, it does not give rise to a peak near the Fermi level. The realization of a spin-3/2 Kondo effect points to the multichannel character of graphene. Hence a spin- $1 / 2 \mathrm{impu-}$ rity could in principle give rise to multi-channel Kondo physics and Non-Fermi liquid behaviour.

The Kondo effect can be controlled by a gate voltage which changes the chemical potential and thereby the filling of the $3 d$-levels. The change in the correlations can be observed by a stark change in the spectra as the system is driven out of the Kondo regime and into the mixed valence regime. Another remarkable result is that the Kondo peaks appear to be pinned to the Dirac point of the graphene substrate rather than to the chemical potential when a gate voltage is applied. This last finding is actually in agreement with very recent STM spectroscopy measurements of Co atoms on graphene which find peaks in the $\mathrm{dI} / \mathrm{dV}$ curves that move according to the applied gate voltage $\frac{38}{3}$. But it has not yet been pointed out by other theoretical work considering the dependence of the Kondo effect in graphene on a gate-voltage ${ }^{15,26}$.

\section{Acknowledgments}

We are grateful to Sasha Balatzky, Kostyantyn Kechedzhi and Régis Decker for useful discussions, and to 
Kristjan Haule for providing us with the OCA impurity solver. Part of this research was carried out at the KITP in the program "Towards Material Design with Correlated Electrons". GK was supported by NSF-DMR0906943.

\section{Appendix A: Details of the embedding method}

Here we show how to compute the embedding selfenergy $\boldsymbol{\Sigma}_{\mathrm{H}}$ given in eq. (11) that describes the dynamic hybridization of the central region $\mathrm{C}$ with the rest of the system (i.e. the graphene sheet). First, we obtain an effective mean-field description of the host material from a GGA calculation for a supercell of clean graphene corresponding to the $\mathrm{C}$ region. From the Kohn-Sham Hamiltonian $\mathbf{H}_{\mathrm{H}}(\vec{k})$ of the host material in reciprocal space we calculate the $k$-dependent Green's function:

$$
\mathbf{G}_{\mathrm{H}}(\vec{k}, \omega)=\left(\omega+\mu-\mathbf{H}_{\mathrm{H}}(\vec{k})+i \eta\right)^{-1}
$$

The local KS Hamiltonian of the supercell is obtained by summing up $\mathbf{H}_{\mathrm{H}}(\vec{k})$ over all k-points within the Brillouin zone:

$$
\mathbf{H}_{\mathrm{H}, 0}=\sum_{\vec{k}} \mathbf{H}_{\mathrm{H}}(\vec{k})
$$

Similarly, the local Green's function of the supercell is given by summing up $\mathbf{G}_{\mathrm{H}}(\vec{k}, \omega)$ over all k-points within the Brillouin zone:

$$
\mathbf{G}_{\mathrm{H}, 0}(\omega)=\sum_{\vec{k}} \mathbf{G}_{\mathrm{H}}(\vec{k}, \omega)
$$

On the other hand, we can write the local Green's function also in terms of the local Hamiltonian $\mathbf{H}_{\mathrm{H}, 0}$ of the supercell and the embedding self-energy (which describes the dynamic hyrbidization with the rest of the graphene) as: $\mathbf{G}_{\mathrm{H}, 0}(\omega)=\left[\omega+\mu-\mathbf{H}_{\mathrm{H}, 0}-\boldsymbol{\Sigma}_{\mathrm{H}}(\omega)\right]^{-1}$. Hence the embedding self-energy is given by:

$$
\boldsymbol{\Sigma}_{\mathrm{H}}(\omega)=\omega+\mu-\mathbf{H}_{\mathrm{H}, 0}-\left[\mathbf{G}_{\mathrm{H}, 0}(\omega)\right]^{-1}
$$

\section{Appendix B: The NCA and OCA impurity solvers}

The general multi-orbital Anderson impurity model can be written in the following form:

$$
\begin{aligned}
\hat{\mathcal{H}} & =\sum_{\alpha \beta} \epsilon_{\alpha}^{d} \hat{d}_{\alpha}^{\dagger} \hat{d}_{\alpha}+\frac{1}{2} \sum_{\alpha \beta \gamma \delta} U_{\alpha \beta \gamma \delta} \hat{d}_{\alpha}^{\dagger} \hat{d}_{\beta}^{\dagger} \hat{d}_{\gamma} \hat{d}_{\delta} \\
& +\sum_{k \nu \alpha \sigma}\left(V_{k \nu \alpha} \hat{c}_{k \nu}^{\dagger} \hat{d}_{\alpha}+V_{k \nu \alpha}^{*} \hat{d}_{\alpha}^{\dagger} \hat{c}_{k \nu}\right)+\sum_{k \nu \sigma} \epsilon_{k \nu} \hat{c}_{k \nu}^{\dagger} \hat{c}_{k \nu}
\end{aligned}
$$

where in order to keep the notation simple we have combined the spin- and orbital degrees of freedom into one index for each impurity level $\alpha$ and each band $\nu$.
The Non-Crossing Approximation (NCA) and the One-Crossing Approximation (OCA) both solve the Anderson impurity model by expansion in the hybridization strength around the atomic limit. The starting point is an exact diagonalization of the impurity subspace, i.e. of the Co $3 d$-shell in our case, including the Hubbard-like interaction term:

$$
\begin{aligned}
& \hat{\mathcal{H}}_{d} \equiv \sum_{\alpha \beta} \epsilon_{\alpha}^{d} \hat{d}_{\alpha}^{\dagger} \hat{d}_{\alpha}+\frac{1}{2} \sum_{\alpha \beta \gamma \delta} U_{\alpha \beta \gamma \delta} \hat{d}_{\alpha}^{\dagger} \hat{d}_{\beta}^{\dagger} \hat{d}_{\gamma} \hat{d}_{\delta} \\
& \stackrel{\text { diag. }}{\longrightarrow} \\
& \sum_{m}|m\rangle E_{m}\langle m|
\end{aligned}
$$

where $|m\rangle$ are the many-body eigenstates of $\hat{\mathcal{H}}_{3 d}$ and $E_{m}$ the respective eigen energies.

One now introduces auxiliary fields $\hat{a}_{m}, \hat{a}_{m}^{\dagger}$ (called pseudo-particles) such that each impurity state is represented by a corresponding pseudo-particle:

$$
\hat{a}_{m}^{\dagger}|\mathrm{PPV}\rangle \equiv|\mathrm{m}\rangle
$$

where $|\mathrm{PPV}\rangle$ is the pseudo-particle vacuum. The completeness of the impurity eigenstates imposes the following constraint:

$$
Q \equiv \sum_{m} \hat{a}_{m}^{\dagger} \hat{a}_{m}=1
$$

The physical electron operators $\hat{d}_{\alpha}^{\dagger}$ can now be expressed by the PP operators:

$$
\hat{d}_{\alpha}^{\dagger}=\sum_{n, m}\left(F^{\alpha \dagger}\right)_{n m} \hat{a}_{n}^{\dagger} \hat{a}_{m}
$$

where $\left(F^{\alpha \dagger}\right)_{n m} \equiv\left\langle n\left|\hat{d}_{\alpha}^{\dagger}\right| m\right\rangle$ are the matrix elements of the impurity-electron creation operator. For later convenience we also define the corresponding matrix elements of the impurity-electron destruction operator as: $\left(F^{\alpha}\right)_{n m} \equiv\left\langle n\left|\hat{d}_{\alpha}\right| m\right\rangle$. The anti-commutation rules for the physical electron operators then require that the PP $\hat{a}_{m}$ is a boson (fermion) if the corresponding state $|m\rangle$ contains an even (odd) number of electrons.

In the PP representation we can now rewrite the Hamiltonian of the generalized Anderson impurity model as follows:

$$
\begin{aligned}
\hat{\mathcal{H}} & =\sum_{m} E_{m} \hat{a}_{m}^{\dagger} \hat{a}_{m}+\sum_{k \nu} \epsilon_{k \nu} \hat{c}_{k \nu}^{\dagger} \hat{c}_{k \nu}+\lambda(Q-1) \\
& +\sum_{\substack{m n \\
k \nu \alpha}}\left(V_{k \nu, \alpha} \hat{c}_{k \nu}^{\dagger} \hat{a}_{m}^{\dagger}\left(F^{\alpha}\right)_{n m} \hat{a}_{n}+H . c .\right)
\end{aligned}
$$

where we have included the constraint $Q \equiv 1$ into the Hamiltonian. The corresponding Lagrange multiplier $\lambda$ can be interpreted as a (negative) chemical potential for the PPs.

In the PP picture, the hybridization with the bath electrons given by the last term in eq. (B6) becomes now the interaction for the PPs. Because of the fermionic and 

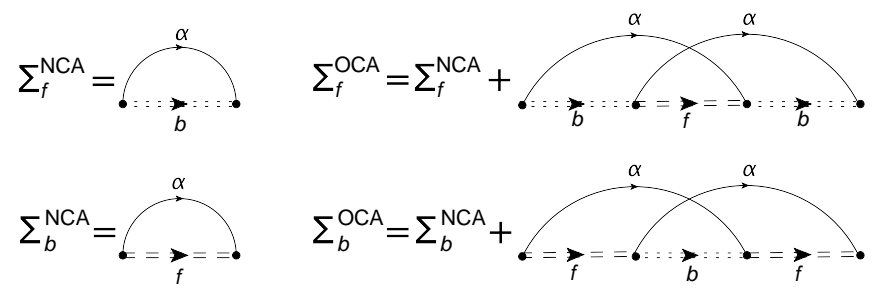

FIG. 7: Diagrams for pseudo-particle self-energies in NCA and OCA approximations.

bosonic commutation rules for the PPs, one can now develop a diagrammatic perturbation expansion in the $\mathrm{PP}$ interaction. The PP propagators can then be written as

$$
G_{m}(\omega)=\left(\omega-\lambda-E_{m}-\Sigma_{m}(\omega)\right)^{-1}
$$

where $\Sigma_{m}$ is the PP self-energy describing the dynamic interaction with the other PPs.

The Non-Crossing Approximation consists in taken into account the diagrams shown in the first row of Fig. 7 for a certain pseudo-particle. The NCA diagrams describe processes where a single electron (hole) jumps from the bath to the impurity and back thereby tem- porarily creating a $\mathrm{PP}$ with $\mathrm{N}+1(\mathrm{~N}-1)$ electrons. The NCA equations correspond to a self-consistent perturbation expansion to lowest order in the hybridization function $\Delta_{\alpha}(\omega) \equiv \sum_{k, \nu} V_{k \nu, \alpha}^{*} V_{k \nu, \alpha}$. Since the fermionic selfenergies depend on the dressed bosonic propagators, and vice versa, the NCA equations have to be solved selfconsistently. Once the NCA equations are solved the physical quantities can be calculated from the PP selfenergies.

OCA takes into account second order diagrams where two bath electron lines cross as shown in the right column of Fig. 7. The self-energies for the fermions (bosons) again depend on the full propagators for bosons (fermions), and hence the OCA equations have to be solved self-consistently. The explicit expressions for the OCA self-energies are second in the bath hybridization function $\Delta_{\alpha}$. The OCA is the lowest order self-consistent approximation that is exact up to first order in the hybridization $\Delta_{3 d} \propto V^{2}$ for all physical quantities while $\mathrm{NCA}$ is only exact to first order in $\Delta_{3 d}$ in the PP and conduction electron self-energies. Further details of the NCA and OCA impurity solver can be found e.g. in Refs. 34,35 .
${ }^{1}$ K. S. Novoselov, A. K. Geim, S. V. Morozov, D. Jiang, Y. Zhang, S. V. Dubonos, I. .V. Grigorieva, and A. A. Firsov, Science 306, 666 (2004).

${ }^{2}$ K. S. Novoselov, A. K. Geim, S. V. Morozov, D. Jiang, M. I. Katsnelson, I. .V. Grigorieva, S. V. Dubonos, and A. A. Firsov, Nature 438, 197 (2005).

3 , Y. Zhang, Y.-W. Tan, H. L. Stormer, and P. Kim, Nature 438, 201 (2005).

${ }^{4}$ K. S. Novoselov, E. McCann, S. V. Morozov, V. I. Falko, M. I. Katsnelson, U. Zeitler, D. Jiang, F. Schedin, and A. K. Geim, Nat. Phys. 2, 177 (2006).

${ }^{5}$ C. W. J. Beenakker, Rev. Mod. Phys. 80, 13371354 (2008).

6 A. H. Castro Neto, F. Guinea, N. M. R. Peres, K. S. Novoselov, and A. K. Geim, Rev. Mod. Phys. 81, 109162 (2009).

7 M. A. H. Vozmediano, M. I. Katsnelson, F. Guinea, arXiv: $1003.5179 \mathrm{v} 1$, unpublished.

8 A. C. Hewson, The Kondo problem to heavy fermions (Cambridge University Press, 1993).

9 B. Uchoa, V. N. Kotov, N. M. R. Peres, and A. H. Castro Neto, Phys. Rev. Lett. 101, 026805 (2008).

10 P. O. Lehtinen, A. S. Foster, Y. Ma, A. V. Krasheninnikov, and R. M. Nieminen, Phys. Rev. Lett. 93, 187202 (2004).

11 J. J. Palacios, J. Fernandez-Rossier, and L. Brey, Phys. Rev. B 77, 195428 (2008).

12 J. H. Chen, W. G. Cullen, E. D. Williams, and M. S. Fuhrer, unpublished (2010), arXiv:1004.3373

13 D. Withoff and E. Fradkin, Phys. Rev. Lett. 64, 1835 (1990).

14 M. Hentschel and F. Guinea, Phys. Rev. B 76, 115407 (2007).

15 K. Sengupta and G. Baskaran, Phys. Rev. B 77, 045417 (2008).
16 P.D. Sacramento and P. Schlottmann, Phys. Rev. B 43, 13294 (1991).

17 P.D. Sacramento and P. Schlottmann, Adv. Phys. 42, 641 (1993).

18 D. L. Cox and A. Zawadowski, Adv. Phys. 47, 599 (1998).

19 D. L. Cox and M. Jarell, J. Phys.: Condens. Matter 8, 9825 (1996).

20 C. M. Varma, Z. Nussinovb, and W. van Saarloos, Phys. Rep. 361, 267 (2002).

21 P. S. Cornaglia, G. Usaj, and C. A. Balseiro, Phys. Rev. Lett. 102, 046801 (2009).

22 Z.-G. Zhu, K.-H. Ding and J. Berakdar, unpublished (2009), arXiv:0912.0182v1.

23 L. Dell'Anna, J. Stat. Mech. P01007 (2010).

24 T. O. Wehling, H. .P. Dahal, A. .I. Lichtenstein, M. I. Katsnelson, H. C. Manoharan, and A. V. Balatsky, Phys. Rev. B. 81, 085413 (2010).

25 T. O. Wehling, A. V. Balatsky, M. I. Katsnelson, A. I. Lichtenstein, and A. Rosch, Phys. Rev. B 81, 115427 (2010).

26 M. Vojta, L. Fritz and R. Bulla, unpublished (2010), arXiv:1002.2215

27 V. Madhavan, W. Chen, T. Jamneala, M. F. Crommie, N. S. Wingreen, Science 280, 567 (1998).

${ }^{28}$ N. Néel, J. Kröger, L. Limot, K. Palotas, W. A. Hofer, and R. Berndt, Phys. Rev. Lett. 98, 016801 (2007).

29 L. Vitali, R. Ohmann, S. Stepanow, P. Gambardella, K. Tao, R. Huang, V. S. Stepanyuk, P. Bruno, and K. Kern, Phys. Rev. Lett. 101, 216802 (2008)

30 N. Néel, J. Kröger, R. Berndt, T. O. Wehling, A. I. Lichtenstein, and M. I. Katsnelson, Phys. Rev. Lett. 101, 266803 (2008).

31 D. Jacob, K. Haule, and G. Kotliar, Phys. Rev. Lett. 103, 
016803 (2009).

32 R. Dovesi et al., CRYSTAL06, Release 1.0.2, Theoretical Chemistry Group - Universita' Di Torino - Torino (Italy).

33 G. Kotliar, S. Y. Savrasov, K. Haule, V. S. Oudovenko, O. Parcollet, and C. A. Marianetti, Rev. Mod. Phys. 78, 865 (2006).

${ }^{34}$ K. Haule, C.-H. Yee, and K. Kim, unpublished (2009), arXiv:0907.0195

35 K. Haule, S. Kirchner, J. Kroha, and P. Wölfle, Phys. Rev. B 64, 155111 (2001).

36 A. Grechnev, I. Di Marco, M. I. Katsnelson, A. I. Lichtenstein, J. Wills, and O. Eriksson, Phys. Rev. B 76, 035107 (2007).

37 A. G. Petukhov, I. I. Mazin, L. Chioncel and A. I. Licht- enstein, Phys. Rev. B 67153106 (2003).

38 V. W. Brar, R. Decker, H.-M. Solowan, Y. Wang, L. Maserati, K. T. Chan, H. Lee, C. O. Girit, A. Zettl, S. G. Louie, M. L. Cohen, and M. F. Crommie, unpublished (2010), arXiv:1006.1014v1.

39 Y. Mao, J. Yuan, and J. Zhong, J. Phys.: Condens. Matter 20115209 (2008).

40 M. J. Frisch et al. Gaussian 03, Revision B.01, Gaussian, Inc., Pittsburgh PA, 2003.

41 A. D. Becke, J. Chem. Phys. 98, 5648 (1993).

42 Unfortunately, we were not able to converge hybrid functional calculations with CRYSTAL06 for Co atoms on graphene sheets. 